\title{
Research on Safety monitoring network of the city subway engineering
} \author{
Tao Wang ${ }^{1, \text { a }}$, Yipeng Zhao ${ }^{1, \mathrm{~b}}$, Zhuo Wang
Zhanhua Zan $^{1, \mathrm{f}}$ \\ ${ }^{1}$ School of Mechanical Engineering, Hebei University of Technology, China \\ ${ }^{2}$ College of Mechanical and Electrical Engineering, Harbin Engineering University, China \\ awtao_1@163.com, ${ }^{\text {b }}$ fengyuzhe13@163.com, ' ${ }^{\mathrm{c}}$ wangzhuo@163.com, ${ }^{\mathrm{d}}$ nanlun@163.com, \\ eyuzhenjiang@163.com, 'zanzhanhua@163.com
}

Keywords: subway engineering, safety monitoring, Internet of things, RFID.

\begin{abstract}
For the monitoring of subway construction and operation safety, early warning and emergency measures, this paper studies the Internet of things(IoT) on safety monitoring of city subway project. This study also include the active smart sensors of city subway IoT and the wireless network composited by tags. This paper researches on the design of tunnel and bridge monitoring sensor on cluster detection. The information nodes of composition sensor format smart labels by connecting RFID through a IoT intermediate. The information reaches the final goal through the multiple hop transmission of smart labels. The monitoring information is transferred by wireless sensor following IoT protocol. So, in this way, achieving the goal of subway tunnel and bridge safety monitoring, early warning and emergency treatment.
\end{abstract}

\section{Introduction}

With the social progress and rapid development of transport services, the number of subway tunnels, cross-river tunnel, bridge engineering increases several times over, the building scale is bigger, and the structure is more complex[1 3]. However, in recent years, tunnels and bridges accidents occur frequently, doing harm to people's lives and property. Monitoring to the construction of tunnels and bridges, the use of monitoring, early warning and emergency measures is very urgent[4].

Urban subway can be classified into two categories, the subway station and subway tunnel, according to the basis of underground building structure. The subway station belongs to large underground caverns including two categories, cavern excavation and open cut construction. Urban subway engineering safety monitoring includes underground caverns, tunnels and foundation pit monitoring[5].

\section{Urban subway engineering safety monitoring IoT system}

City subway safety monitoring content: city subway is built in the surroundings of ground stress field and natural rock consisting of various surface structure. Therefore, the engineering security depends largely on the mechanical properties of the surrounding rock itself and self-stability and the comprehensive characteristics of its support. As the city subway buried in the underground certain depth, the natural geologic materials exist in joint fissure, stress and groundwater, the construction of the city subway is much more complex than the ground subway project, especially before the city subway excavation, its geological conditions, rock forms and other information that is not easy to grasp, the mechanical parameters that is difficult to determine. Therefore, on-site safety monitoring is an effective way to obtain factual information of the change of building traits and will transfers the real-time data feedback to the project management[6,7]. So on-site safety monitoring will play a crucial role on the project of safety construction and operation.

In accordance with the foregoing safety monitoring principles and project of urban subway, establishing active smart sensor based on IoT and wireless network which is composed of labels. The 
network consists of the afore mentioned tunnel and bridges monitoring sensors which cluster monitor points. Sensor nodes and RFID are connected by an IoT intermediate to form smart labels, and the information through multihop transmission between smart tags until reaching the ultimate goal[8,9]. The transfer of the information monitored uses a wireless sensor network protocol, so as to monitor the safety of subway tunnels, bridges and get early warning and emergency response.

Core technology of IoT is the sensor, radio frequency identification technology (RFID), wireless sensor networks and data fusion treatment technology[10]. In accordance with the provisions of the agreement to achieve the goal that things and things, people and people, people and things connect at any time. Thereby, this system realizes data exchange and communication, becoming a smart identification, location tracking, monitoring and management huge network system.

The traditional tunnel, bridge engineering safety monitoring is given priority to artificial measuring or half automation measurement, and the drawback is obvious that it is unable to obtain information of critical moment and it is cost manpower. IoT monitoring uses sophisticating detection sensing equipment and wireless transmission technology to improve the disadvantage of the traditional monitoring way[11]. Its basic structure is the sensor cluster which forms the frontend sensing network through wireless networking module. The system adopts wired or wireless which will connects sensor group into the net by IoT intermediate because the construction area or volume of tunnels and bridges is relatively large. The sensor group is placed on a monitoring point, clearly defining borders norms of safe state, predicting unsafe time and status, and proposing security solutions. These conventions will be quantized. Then it will be stored in the system after the establishment of system model. The system compares to alarm and take emergency measures automatically.

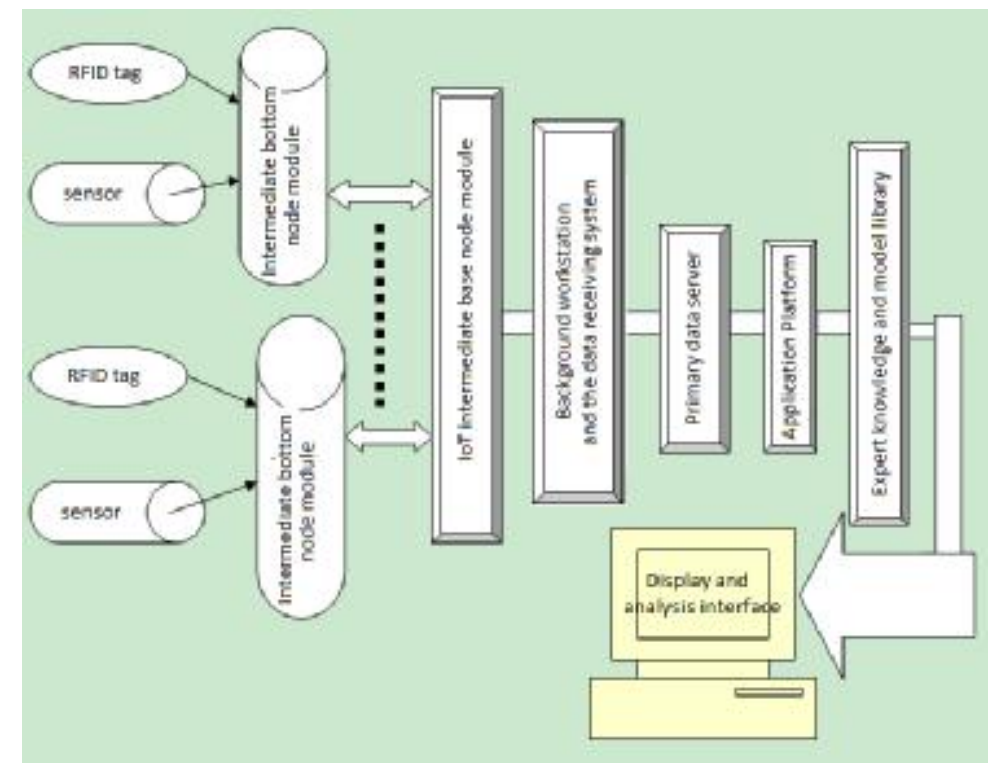

Fig. 1 Tunnel and bridge safety monitoring on IoT

\section{Key technology of urban subway IoT systems}

\subsection{Zigbee technology application}

Zigbee is synonymous of IEEE 802.15 .4 protocol. According to this protocol, this technology is a short-range, low-power wireless communications technology. Its characteristics are close range, low complexity, self-organization, low power, low data rate and low cost, mainly suitable for automatic control and remote control field. ZigBee uses the $2.4 \mathrm{GHz}$ frequency range, using frequency hopping and spread spectrum technology, and it can be networked with 254 nodes. In short, ZigBee is an inexpensive, low-power short-range wireless network communication technology. 
Zigbee protocol structure system is made up of senior application specification, convergence layer, network layer, data link layer and physical layer. IEEE 802.15.4 working group is mainly responsible protocol of the physical layer and MAC sub-layer, the rest of the agreement mainly refer to the existing standards of traditional wireless technology.

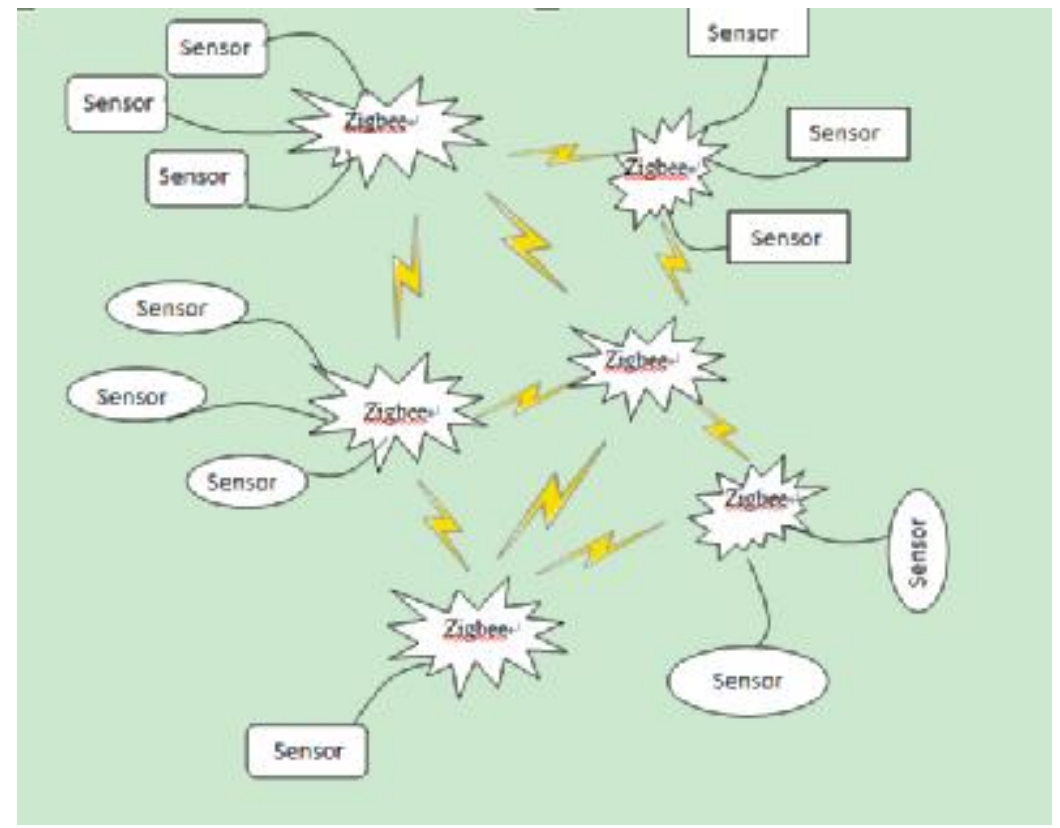

Fig. 2 RFID tagged by wireless network formed front sensor network

The smart sensing tags used in the monitoring of tunnel and bridge are smaller, lower cost and the network data traffic is relatively small, so this system chooses IEEE802.15.4 / ZigBee network protocol as the smart sensor tags. ZigBee protocol may improves the RFID tags that have monotonic function and small communication range to being sensor nodes. The smart tags in the system will cause a lot of point to point security assurance issues, and the IEEE802.15.4 protocol provides the appropriate key encryption technology and authentication to ensure the security of the whole system.

\subsection{RFID and smart tag technology}

The target recognition is realized by the Radio frequency signal automatically, and its information will be executed signs, registered and managed. The system components comprise electronic tag (composed of a chip and tag antenna or coil, to communicate by inductive coupling or electromagnetic reflection principle and reader), the reader (reading or writing tag information of the device) and the antenna (which may be built into the reader, but also can connected the interface of reader antenna by coaxial cable) the principle of Figure 3.

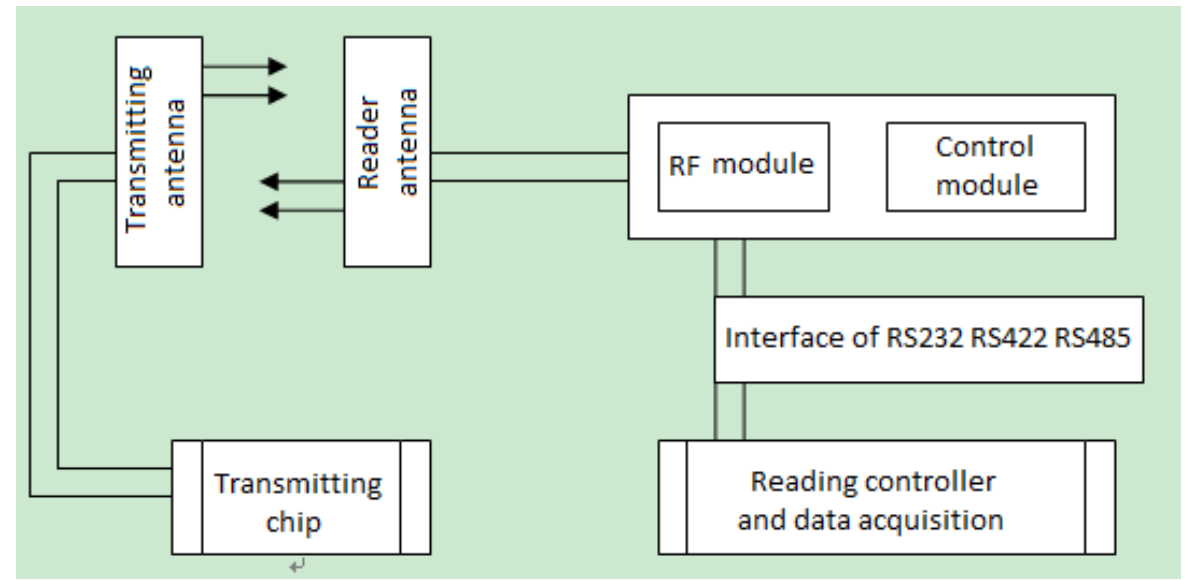

Fig. 3 The working principle of RFID system 
At present, RFID is mainly focused on the identification, it can realize the identification and management of the target. But the RFID systems have limited reading distance, poor immunity and the higher cost to realization; WSN focuses on networking, data transmission, and it is simple to deploy and its cost of realization is low etc. But it generally do not have the node identification function, so the integration of RFID and wireless sensor networks will make up the deficiencies and defects both. Now it has been designed into the sensor active RFID tags or semiactive tags, because active tags similar to wireless sensor network nodes and they do not realize the wireless sensor network node functions fully. So a microprocessor is configured to active tags and the active tags can compare with the phase node in wireless sensor network. In other words, the wireless sensor node can be replaced by the active tags. This RFID application system for wireless sensor nodes is called smart sensor tags.

This tag is similar to the wireless sensor node. This system can pass data through multihop manner, and pass the final data to sink node, equivalent to a network base station. The entire network requires only one reader, reducing the number of readers and wired network equipment effectively. Smart sensor tags are similar to the nodes of wireless sensor network, they not only have data processing capabilities of wireless sensor network nodes, but also have the ID number of FRID tags. In this way, this system achieves the identification function through the wireless transmission network. Combined with the advantages of the two technologies, the two technologies complement each other defects in the future detection of tunnels, bridges and other major projects and brings more powerful advantage.

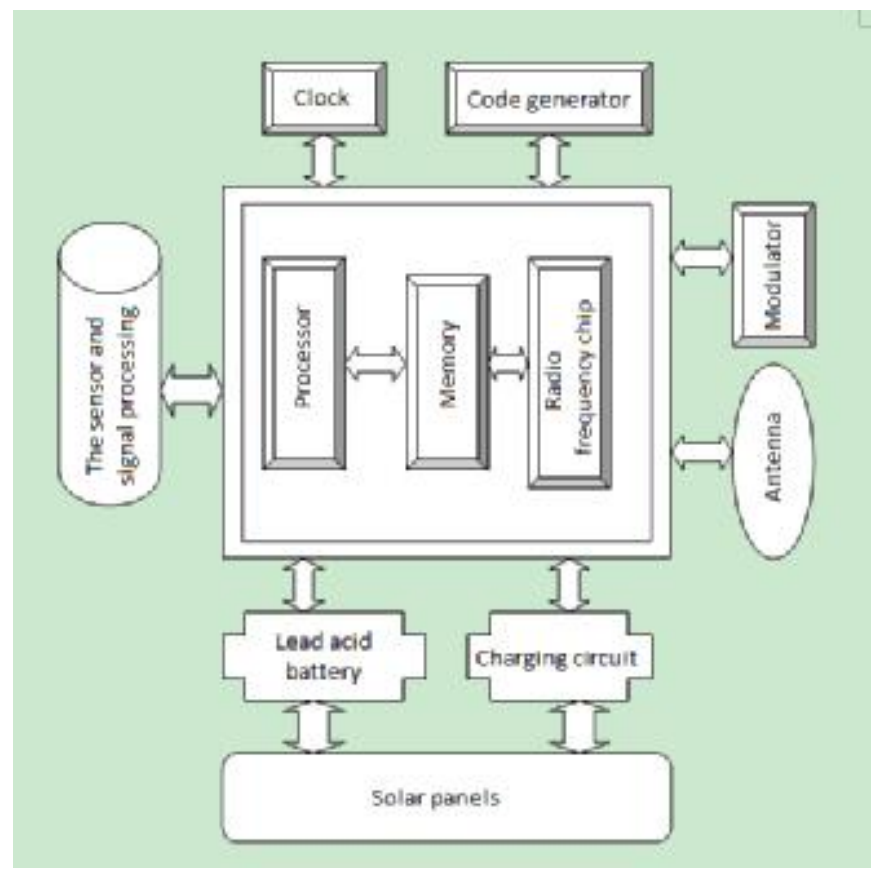

Fig. 4 The connecting body structure of middle smart label

\subsection{Multichannel data acquisition technology}

Using Dual CPU control, the slave CPU is responsible for collecting seven road data at the same time and response of the master CPU commands. The master CPU processes data, data display, keyboard input, alarming system, voltage value of voice broadcast channel. The master CPU communicates with the slave CPU by international standard RS485 differential mode interface of which communication speed and distance are larger than a standard RS232 interface mode, and at least lines (just two). It becomes a kind of data acquisition system with voice broadcast and high-performance, highly smart and practical long-range multichannel.

\section{Formation of IoT system}


This Internet of things system is built mainly for tunnel and bridge. The system uses the general middle connecting body circuit module directly with the interface to common displacement sensor, pressure sensor, direct interface of radio frequency identification, direct current interface Ethernet system and internet. Finally it will integrate smart networking system in tunnel and bridge.

The common sensors include convergence gauge, precision level gauge, multi-point displacement meter, crack meter, acoustic instrument, dynamometer, bolt stress, stress meter, strain gauge, accelerometer, speed meter, weir and thermometer. The other sensors have the tunnel safety information monitoring sensor, GPS satellite positioning device, construction machinery safety information sensor, video monitoring unit, dangerous goods status information monitoring sensor and so on.

Radio frequency identification consists of sensor types and location information. It also includes a shield machine, frame and load information system.

The network and computer smart software contain the GPS satellite positioning and optimization path information of construction machinery with intelligent and tips, the safety of the tunnel intelligent information and warning, monitoring of safety in construction of intelligent information and tips, dangerous situation of intelligent information monitoring and warning, monitoring system, smart video material anti-theft vehicle tunnel accident monitoring smart video system.

The general middle connector module system mainly includes: data interface module, memory module, microcontroller, RF transceiver module, GSM module, power management module and so on. They are integrated in a single block of double circuit board, composed of general middle connecting body module. The intelligent control is composed of a middle connecting body module in the MCU and combines with the upper smart computer software to complete the process, and configures the vehicle mounted receiver to form a complete IoT system.

In the typical application structure, data interface module uses MAX125 as multichannel data acquisition chip, MSP430F149 as MCU, IDT7132 as storage module with dual port RAM, CC2420 as RF transceiver module. Circuit board is double hybrid integrated circuit board with input, output and general data port.

The scheme of Internet of things technology is integrated data interface module, memory module, microcontroller, RF transceiver module, GSM module and power management module in a single block of double hybrid integrated circuit board. The circuit board connects each other by the wireless/ wired transmission communication. The circuit board is set up with universal input, output, data ports and power switch, integrated to form the connecting body module common intermediate, reconfiguration of SCM and the upper computer intelligent control software, completing the networked system composition.

Through the Internet of things universal port directly with the general sensor interface link, connecting body module of common intermediate and the radio frequency identification (RFID) interface directly, connecting the existing Ethernet system and Internet links directly, then upgrade to form a smart networking system directly.

\section{Implementation of security monitoring IoT system of city subway engineering}

\subsection{Scheme demonstration}

Design and demonstration of the overall scheme of security monitoring IoT system of city subway engineering. It includes: the overall layout, the determine of monitoring program, sensor selection and settings, the intermediate base station layout, wireless transmission setting, mobile base station layout, site monitoring base station layout, the remote control center settings and so on.

\subsection{Design and budget of IoT system of city subway engineering}


It includes obtaining geological and environmental data, such as tunnel engineering geological and hydrogeological data, the plane, vertical and horizontal sections and surrounding rock classification data, including: 1) the geological report (including longitudinal and cross section, the bore histogram and plane display chart); 2) the statistical structure of geological data, fracture diagram; 3) classification of surrounding rock, the shape of weak structure plane; 4) earthquake intensity; 5) the distribution of groundwater; 6) cavern stability evaluation.

Geological parameters experiments includes: 1) rock and surrounding rock mechanical test parameters; 2) mechanical parameters of weak structural surface; 3 ) model test report.

This engineering also include the design of all IoT system relevant structures, reserve civil structures and engineering structures, the construction of IoT system ,the stage of acceptance and the overall acceptance.

\section{Conclusions}

This paper first introduces the importance of urban subway safety monitoring and the necessity of using IoT system. The key technologies of IoT system include the application of ZigBee technology, RFID and smart tag technology, multichannel data acquisition technology. These key technologies are connected closely and complement each other, and they complete the monitoring of urban subway together. Finally, this paper lists the specific IoT scheme from details to realize the real-time monitoring of urban subway.

\section{References}

[1] Costa F J, Noble A G, The growth of metro systems in Madrid, Rome and Athens. Cities. 7(3) (1990) 224-229.

[2] Knowles R D, Light rail transit development in Britain. Geography. 77(1) (1992) 17-21.

[3] Friedman M, Cordovil C, The Rio de Janeiro subway system: A case study in applying theory to practice. Transportation Research Part A. 13(2) (1979) 125-130.

[4] Deng Xiaopent, Li Qiming, Zhou Zhipeng, Statistical Analysis of Safety Events Patterns in Metro Construction. Statistics and Decision. 9 (2010) 87-89.

[5] Jin Lei,Peng Jian,Peng Fangle, Sorting Theory and Investigation of Urban Metro Station. Chinese Journal of underground space and Engineering. 2010.

[6] Michael D, Urban geology of Canadian cities. Canadian Journal of Urban Research. 11(2) (2002) $1-365$.

[7] Haworth R J, The shaping of Sydney by its urban geology. Quaernary International. 103(1) (2003) 41-55.

[8] Atzon L, Iera A, Giacomo M, The Internet of Things: a survey. Computer Networks. 54(15) (2010) 2787-2805.

[9] Information technology-AIDC techniques-RFID for item management Air interface. ISO/IEC 18000. 2004.

[10] Medaglia C M, Serbanati A, An overview of privacy and security issues in the internet of things. The Internet of Things. 2010.

[11] Mattern F, Floerkemeier C, From the Internet of computers to the Internet of things. From Active Data Management to Event-Based Systems and More. 2010. 